\title{
LA FORMACION DE UN SISTEMA \\ TRANSNACIONAL EUROPEO \\ DE TRANSPORTES Y COMUNICACIONES \\ EN LOS SIGLOS XIX Y XX
}

JAVIER VIDAL OLIVARES

Universidad de Alicante

Durante los días 16 al 19 de mayo de 1993 tuvo lugar en San Miniato (Toscana, Italia) la preconferencia de la sesión B del XI Congreso Internacional de Historia Económica bajo el título de European networks: New Approaches to the Development of a Transnational Transport and Communications System (XIXth and $x x t h$ Centuries). Como es conocido, las denominadas reuniones B de los Congresos de la Asociación Internacional de Historia Económica tienen como objetivo central el fomento de la coordinación investigadora de amplias áreas en las que los trabajos realizados o en marcha son relativamente numerosos, abarcando además una amplia nómina de investigadores de varios países. Este ha sido, fundamentalmente, el interés de los organizadores de la sesión: los profesores A. Carreras, A. Giuntini y la profesora M. Merger.

Desde el punto de vista formal, las reuniones que se llevaron a cabo en el centro I Capuccini de la Cassa di Risparmio di San Miniato trataron de recoger todos los sistemas de transporte ya fuesen ferrocarriles, líneas maritimas, relaciones aéreas, oleoductos y gasoductos, o bien sistemas de telecomunicación, como los telégrafos. Los temas abordados lo fueron desde metodologías y objetivos diferentes, si bien los ferrocarriles fueron el sistema de transporte que recogió buena parte de las comunicaciones mientras que la navegación marítima apenas recibió atención. En el caso holandés fue W. Van den Broeke quien planteó en su trabajo cómo los ferrocarriles de los Países Bajos, trazados a través de las fronteras alemanas, buscaban tanto el hierro procedente de las regiones del Rhin como el carbón prusiano y alemán en general, a fin de favorecer el comienzo de la mecanización en los diversos sectores de la industria holandesa. También Prusia y los Estados alemanes, por su parte, trataron de imponer la construcción de ferrocarriles para conseguir una unión rápida y compe- 
titiva, mediante varias líneas de ferrocarril, para alcanzar el mar. Por tanto, el papel de Alemania apoyando la construcción de los ferrocarriles fue fundamental en los trazados holandeses. En el caso de este último pais, según el trabajo presentado por D. Ziegler, las líneas férreas fueron construidas por los diferentes Estados sin practicar una planificación previa. Esto determinó finalmente una particular forma de competición con otras formas de transporte, tales como los fluviales y los que se realizaban a través de las carreteras, al menos hasta la unificación aduanera del Zollverein y la todavía más posterior unidad nacional alemana de finales del siglo XIX.

La Europa oriental contó, por su parte, con varios trabajos en los que se abordaba la estructuración histórica de la red de transportes. Uno de los más destacados fue el que presentaron los profesores Z. Landau y W. Morawski al ocuparse de analizar la influencia de los factores políticos sobre el desarrollo de la red de transportes en Polonia, Lituania, Bielorrusia y Ucrania desde finales del siglo xviII. Sin duda la conclusión más relevante de este trabajo, tras estudiar y explicar todas las relaciones económicas que se han ido establecien. do en estos territorios hasta tiempos recientes, es que, al contrario de lo ocurrido en la Europa occidental -en la que los cambios de fronteras por razones políticas no han sido demasiado trascendentes-, en el área oriental la frecuencia en las modificaciones y alteraciones fronterizas, por razones incluso estratégicas de índole militar, han influenciado mucho más y en sentido negativo las formas que ha ido adquiriendo la red de transportes, sin que los factores económicos hayan desempeñado el papel fundamental.

Desde un enfoque teórico hay que destacar aportaciones significativas al papel que ha desplegado el ferrocarril como instrumento uniformizador en la difusión de nuevas tecnologias en el sector transportes. Los trabajos de D. J. Puffert y E. Cefis trataban de abordar, desde perspectivas diversas, los problemas que los ferrocarriles europeos han tenido y tienen que afrontar para unificar la explotación de sus redes ferroviarias. La primera de las investigaciones, realizada desde una perspectiva actual, pone de relieve cómo el ancho de vía, los diferentes sistemas eléctricos y las especificaciones de los nuevos trazados de líneas de alta velocidad son problemas que deben ser abordados calculando los costes que su conversión en sistemas estandarizados para toda Europa representaría y si éstos superarian los beneficios derivados de la integración. Con una perspectiva netamente norteamericana se señala que los problemas que se esconden tras este deseable proceso de uniformización técnica son principalmente aquellos que aparecen asociados a las rivalidades tecnológicas que están detrás de la posible internacionalización de la red europea, tal y como se ha puesto en evidencia con las pugnas franco-alemanas por el desarro- 
llo de las redes de alta velocidad en Europa, que han afectado a algunos países del continente, entre ellos España.

Es precisamente E. Cefis la que analiza este ultimo problema de forma monográfica. Entre sus aportaciones más significativas al problema de la homogeneidad de la tecnología ferroviaria de la futura red de Alta Velocidad europea esta autora destaca el hecho de que deben ser las instituciones europeas y no sólo las fuerzas del mercado las que deben contribuir a la estandarización del sistema tecnológico de la futura red. Empresas proveedoras y compañías ferroviarias deberian llevar a cabo su cometido a través de las regulaciones necesarias y utilizando financiación de la Comunidad Europea.

Otras intervenciones significativas sobre historia ferroviaria, en tanto que modo de transporte mayoritario hasta la década de 1920, se centraron en el fracaso de la unidad ibérica mediante el ferrocarril, cuya comunicación corrió a cargo de M. Pinheiro; sobre el despegue comercial de la red férrea suiza gracias al impulso del turismo entre Inglaterra y el pais alpino, estudiado por $\mathrm{L}$. Tissot; acerca de los problemas de origen histórico de la red de transportes transpireinaica, expuestos en la comunicación de A. I. Escalona; en torno a los problemas de la internacionalización de la red ferroviaria y las estrategias comerciales de las compañias españolas a cargo de J. Vidal, y, finalmente, el trabajo de investigacion de A. Schram, en donde se reconstruyen los tráficos de las redes ferroviarias del norte de Italia a fines del siglo XIX. Lo más destacado de este último trabajo lo constituye la idea de que la apertura del túnel de San Gotardo entre Italia y Suiza significó una aportación sustancial a los tráficos italianos, aunque el grado de apertura de la red (definido como el flujo total exportador e importador respecto del tráfico total de la red) con otros países europeos - singularmente Francia y Austria - ya era bastante importante. En todo caso, antes de 1890 la estructura del tráfico de los ferrocarriles en el área septentrional italiana parecía reflejar una «exportación» básicamente agraria, frente a una «importación» preferentemente industrial.

Un elemento importante y novedoso en este último trabajo mencionado es la utilización de mapas computerizados, los cuales permiten cartografiar la información estadística disponible. Es precisamente esta herramienta informática la base de las comunicaciones presentadas por A. Carreras con A. Giuntini y la de A. Kunz. La primera versó sobre la creación de un atlas de los ferrocarriles italianos, mientras que la segunda se centró en la reconstrucción de los tráficos internacionales desarrollados a través de los canales en Alemania. Por su parte, el trabajo de los primeros se basa en la utilizacion de un nuevo software, el Atlas.Gis, que permite, utilizando una matriz de datos, la reconstrucción mediante el ordenador de los diferentes estados que presenta una red de transporte a 
lo largo del tiempo. Aunque su objetivo central es avanzar en un proyecto de investigación más ambicioso en el que se estudian las relaciones entre el crecimiento económico y las redes de transporte y comunicación a escala europea, en esta primera aproximación el objetivo es de menor alcance: poner de manifiesto los problemas de fuentes existentes en Europa para conseguir una base de datos con la que reconstruir el ritmo de avance de las redes de transporte. Con estas limitaciones, la primera fase de la investigación se limita al caso italiano y sólo teniendo en cuenta los ferrocarriles y carreteras. En todo caso, los resultados de esta comunicación permiten augurar un buen futuro en la investigación y poder alcanzar de este modo conclusiones importantes acerca de la idea de redes transnacionales de comunicación, concepto éste que va más allá de los tradicionales estudios de relaciones internacionales o de creación de una estructura de empresas tipicamente multinacionales.

Con un sistema diferente pero con similares objetivos a los del trabajo de A. Carreras y A. Giuntini, es necesario referirse a la comunicación de A. Kunz. Este autor utiliza un programa de ordenador para cartografiar los tráficos de mercancias que el Imperio alemán realizó con los mercados exteriores a través de las fronteras que representaban sus amplias y extensas redes de canales durante la segunda mitad del siglo xix. La principal conclusión es que en el transporte interfronteras de Alemania con el centro de Europa y los mercados occidentales tuvieron un mayor peso relativo los transportes fluviales que los realizados por ferrocarril. La red de canales desempeñó un papel central en los flujos transnacionales hasta 1914, tal y como un análisis comparativo de medios de transporte revela para el caso germano.

Junto al ferrocarril otros modos de transporte y comunicación recibieron una atención significativa, aunque, por su papel estructurante, históricamente los caminos de hierro recibieran la mayor atención de los investigadores. Así, el transporte aéreo sólo mereció una investigación: la de M. Dierikx y P. J. Lyth, referida ésta al conjunto de Europa entre 1920-1970. En este trabajo se lleva a cabo la construcción de un modelo de análisis de redes típico de la geografía; la construcción de un conjunto de índices (de atracción, de conectividad o aislamiento) a partir de los tráficos aéreos entre aeropuertos europeos con una secuencia cronológica amplia de medio siglo de aviación civil. La investigación revela cómo se ha ido articulando un conjunto de ejes de tráfico aéreo: primero el de Londres-París-Amsterdam (1930) y, después de la II Guerra Mundial, la ampliación del eje: primero con un sentido Este-Oeste y más tarde claramente Norte-Sur, ya en la década de 1960. La red aérea se fue desarrollando haciendo más cerrado y accesible el espacio europeo, por ello la densidad de la red a partir de 1950-1960 reflejó el proceso de reconstrucción 
de la economía europea que, con la expansión del turismo en los sesenta, coadyuvó a difundir pautas de comportamiento similares en la zona occidental del continente.

La constitución de otras redes de comunicación en el marco europeo que encontraron desarrollo en la reunión fueron las del gas. A. Beltrán y J. P. Wi lliot explicaron cómo se ha ido configurando la distribución del gas natural en Europa, especialmente tras los problemas asociados a la difusión de nuevas energías en relación con la crisis industrial de los años setenta. La electricidad mereció también la atención de un investigador francés como $\mathrm{C}$. Bouneau, quien explicó en su comunicación la interconexión de una gran potencia generadora de electricidad como Francia con el resto del continente.

Por la escasez de investigaciones disponibles merecen ser destacados los trabajos que se ocuparon de la evolución histórica del telégrafo como red de comunicación internacional europea y transcontinental. Asi, M. Arcarons planteó en su ponencia los fallidos intentos españoles por convertir la península en la pionera del desarrollo de la comunicación telegráfica por cable entre Europa y América durante la segunda mitad del siglo XIx. Por su parte, los daneses P. Thestrup y H. C. Johansen señalaban cómo Dinamarca se convirtió, a fines del siglo XIX, en el pais que más aprovechó su situación geográfica, claramente intermediadora o de tránsito, entre el norte y el sur. Ello le permitió un impulso considerable al sector de las comunicaciones, lo cual fue aprovechado para desarrollar una clase empresarial que expandió sus compañias mas allá de su pais, mejorando el nivel tecnológico de la industria danesa hasta la I Guerra mundial.

Por su contribución a la fuerte expansión de las redes de transporte y comunicación, el sector turístico mereció algunas comunicaciones centradas en el conjunto de Europa, desde un enfoque global en la ponencia de J. O. Lundgren o abarcando un caso particular como el de las islas Baleares en la aportación de C. Manera y J. M. Escartín. El primero centró su interés en el espacio turístico europeo utilizando los clásicos instrumentos analíticos de los geógrafos, basados en modelos de atracción o expulsión mediante el uso de datos y factores varios como precios, distancia, caracteristicas políticas o infraestructuras disponibles. Lo más significativo para este autor es que la situación en la política internacional desde 1945 ha justificado la creación sólo de un espacio turístico, restringido a la Europa occidental. Pero esto significa que a partir de 1989 sólo podrá haber una apertura de los mercados del este hacia el mercado occidental si crece la disponibilidad de todo tipo de infraestructuras, tal y como ocurrió en la Europa occidental con ferrocarriles, carreteras y aeropuertos en el desarrollo de las áreas septentrionales y meridionales. Por su parte, el 
caso de las Baleares ejemplifica muy claramente la mejora de la accesibilidad de las islas desde 1970 al impulsar, gracias al turismo, el desarrollo de la navegación aérea y marítima.

Pese a su protagonismo en la articulación de la red de transportes europea, los canales no recibieron, sin embargo, toda la atención que estas infraestructuras merecerian de acuerdo con el relevante papel desempeñado en la historia económica del continente. Sólo $M$. Merger presentó una investigación en la que se pasó revista a las vicisitudes históricas del proyecto de unión del río Rhin y del Ródano a través de la construcción de un canal. Desde mediados del siglo XIX hasta $1982 \mathrm{M}$. Merger explica los problemas que impidieron que este eje fundamental, que hubiera significado la unión del norte europeo con el Mediterráneo, llegase a cristalizar. El problema central que aquejó a esta magna obra fue la falta de apoyo de la administración central francesa a una politica de dotación de infraestructuras fluviales que hubiese dado a Francia un sistema plurimodal de transporte similar al alemán, cuestión que tiene sus orígenes históricos en el siglo XIX cuando tuvo lugar la revolución de los transportes.

Aunque no ubicado en el continente europeo, el canal de Suez ha estado vinculado directamente a la expansión económica de los paises del viejo continente y, por ello, mereció la atención de algunas comunicaciones. La más relacionada con el tema central del Coloquio fue la de E. H. Tuma. Este autor repasa la historia del Canal y concluye que la obra debe considerarse como una infraestructura netamente europea. En efecto, el canal sirvió para unir los mercados internacionales fuertemente dominados por las economias del viejo continente, pero especialmente funcionó como un vinculo central entre las colonias de Africa y Asia con sus metrópolis. Los beneficios para Egipto frente a los que la obra y su compañia explotadora reportaron para el consorcio europeo fueron, hasta su nacionalización en 1956, muy reducidos.

El Coloquio también recogió algunos aspectos importantes respecto de los temas centrales a discutir, en los que se trataron de poner de relieve cuestiones teóricas de carácter general, como el papel del sector público en la dotación de infraestructuras o de tecnologias de comunicación; los origenes de la red europea de autopistas vinculado a los regímenes totalitarios del periodo de entreguerras o al papel unificador que las empresas constructoras en su acción internacional han desempeñado en Europa. En todos estos temas mencionados y a la largo de las sesiones fue fundamental la acción de los moderadores -F. Caron, R. Fremdling, H. Morsel, T. Hughes, R. Leboutte, D. Headrick, S. Salsbury-, los cuales dirigieron las intervenciones y debates aportando puntos de vista que mejoraron notablemente las conclusiones globales. Estas servirán 
de punto de partida para la discusión general, que tendrá lugar en Milán en el otoño de 1994. En cualquier caso, lo que puede ya adelantarse es que el Coloquio y las comunicaciones presentadas pusieron de relieve el papel fundamental que las infraestructuras de transporte y las comunicaciones que aquéllas facilitan han desempeñado, tanto en el crecimiento económico como en la articulación del espacio europeo, aunque en este último punto los obstáculos nacionales han sido y todavía son de gran magnitud. En esta línea un enfoque investigador basado en el concepto de transnacionalidad, como el propuesto por A. Carreras y A. Giuntini, centrado en el interés por interpretar en un sentido general el espacio que los diferentes países ocupan teniendo en cuenta las relaciones físicas entre unidades administrativas y buscando un desarrollo económico común, podría ser muy productivo para la investigación en el futuro. El Coloquio de San Miniato contribuyó a estimular futuros trabajos orientados hacia este fin.

\section{COMUNICACIONES PRESENTADAS}

ARCARONS, M.: L Espagne et les liaisons télégraphiques transatlantiques (1858-1898).

Avelar Pinheiro, M.: Histoire d'un divorce: l'intégration des chemins de fer portugais dans le réseau ibéerique.

BARJoT, D.: La mise en place des infraestructures européennes de transport: le rôle des entrepreneurs français de travaux publics (1857-1974).

Beltrán, A., y Williot, J. P.: Développement et modes de gestion du réseau de gaz naturel en

Europe occidentale depuis les années 1960.

Blankart, Ch., y G. Knieps, Market or State in Public Services. A public Service Approach.

BORTOLOTTI, L.: Les premières propositions d'un système europèen d'autoroutes (1926-1937).

Bouneau, C.: La génèse de l'interconnexion éléctrique de la france du début du siècle à 1946.

BruhaCs, J.: Régime international de la navigation sur le Danube.

Carreras, A.: y Giuntini, A.: A cartographical project: notes on the development of a compu terized historical atlas of European transports and communications (XIXth and XXth centuries).

CEFis, E.: Tecbnological Innovation, Standardization and Integration: the case of Hihg-Speed Trains.

Dierikx, M.: y LyTh, P.: The Development of the European air Transport Networks, 1920 1970: An Explanatory Model.

Escalona, A.: The Network of Pyrenean Passages: A History of Forward and Backward Steps.

$\mathrm{F}_{\Lambda \mathrm{RNIE}}$ D.: Transportation Strategies of Oil Companies and Tanker Operators in relation to the Suez Canal, 1929-1989.

Hedjuk, I.: y Filipczyck, J.: Polish Transportation System in Integrated Europe.

KaLINSKI, J.: Common Freight Car Pool-OPW (1964-1990).

Kostov, A.: Les Balkans et le réseau ferroviaire européeen avant 1914. 
Kunz, A.: The Development of a Central European waterway network since 1850.

Landau, Z., y Morawsi, W.: The influence of Political Factors on the Development of Transportation Network in Poland, Litbuania, Bielorrusia and Ukraine from the End of the Xvill century.

Lundgren, J. O.: European Tourist Space and Tourist Travel Networks. Past, Present and Future.

Manera, C., y Escartin, J. M.: The Balearic islands incorporation into the European transport networks, 1850-1985.

MERGER, M.: La liaison Rbin-Rbône ou l'bistorie d'un serpent de mer (1834-1991).

PUFFERT, D.: The Tecbrical Integration of the European Railway Network.

SCHRAM, A.: Cross-border railway networks: Veneto, 1860-1880.

Szfvos, M.: The rise of Networks and the structure of public domain in the 19tb Century.

Thestrup, P.: y Johansen, H. Ch.: Denmark as a Nodal Point in the International Telegraph Network in the 1870's.

Tissot, L.: Développement touristique et développement ferroviaire. Leétablissement d'un résau transeuropéen entre l'Anglaterre et la Suisse (1850-1914).

Tuma, E. H.: Suez Canal: Another dimension in the European Network.

VAN DEN BROEKE, W. Frontier crossing railway traffic and the Duth economy in the second balf of the nineteenth century.

Van der Herten, B., y Verhoest, P.: The Belgian Contribution to the Creation of 19 th Century International Communications Networks.

VIDAL, J.: National or International Markets? Railway Companies in Spain and their relations with France and Portugal, 1850-1914.

ZIEGLER, D.: Particularism as a determining factor in the development of the German iransport network in mid-ninenteenth century. 